\title{
Corrigendum: A Perspective on the Success and Failure of BCG
}

\author{
Pawan Kumar* \\ Department of Preventive Oncology, Dr. B. R. Ambedkar Institute Rotary Cancer Hospital, All India Institute of Medical \\ Sciences, New Delhi, India
}

\section{OPEN ACCESS}

Edited and reviewed by: Juraj Ivanyi,

King's College London,

United Kingdom

*Correspondence: Pawan Kumar pksbio30@gmail.com

Specialty section:

This article was submitted to Vaccines and Molecular Therapeutics,

a section of the journal

Frontiers in Immunology

Received: 07 January 2022

Accepted: 12 January 2022

Published: 09 February 2022

Citation:

Kumar P (2022)

Corrigendum: A Perspective on the Success and Failure of BCG.

Front. Immunol. 13:850325. doi: 10.3389/fimmu.2022.850325
Keywords: tuberculosis, BCG, vaccine efficacy, immune response, environmental mycobacteria, heterogeneity, geographical latitude

\section{A Corrigendum on}

A Perspective on the Success and Failure of BCG

by Kumar P (2021). Front. Immunol. 12:778028. doi: 10.3389/fimmu.2021.778028

In the original article, there was an error in Figure 1 as published. Specifically, the curve representing the host anti-Mycobacterium tuberculosis $(\mathrm{Mtb})$ immune response in unvaccinated people in low environmental mycobacteria $(\mathrm{EMb})$ abundance areas (normal red line) was not drawn correctly. The corrected Figure 1 appears below.

The authors apologize for this error and state that this does not change the scientific conclusions of the article in any way. The original article has been updated.

Publisher's Note: All claims expressed in this article are solely those of the authors and do not necessarily represent those of their affiliated organizations, or those of the publisher, the editors and the reviewers. Any product that may be evaluated in this article, or claim that may be made by its manufacturer, is not guaranteed or endorsed by the publisher.

Copyright $\odot 2022$ Kumar. This is an open-access article distributed under the terms of the Creative Commons Attribution License (CC BY). The use, distribution or reproduction in other forums is permitted, provided the original author(s) and the copyright owner(s) are credited and that the original publication in this journal is cited, in accordance with accepted academic practice. No use, distribution or reproduction is permitted which does not comply with these terms. 


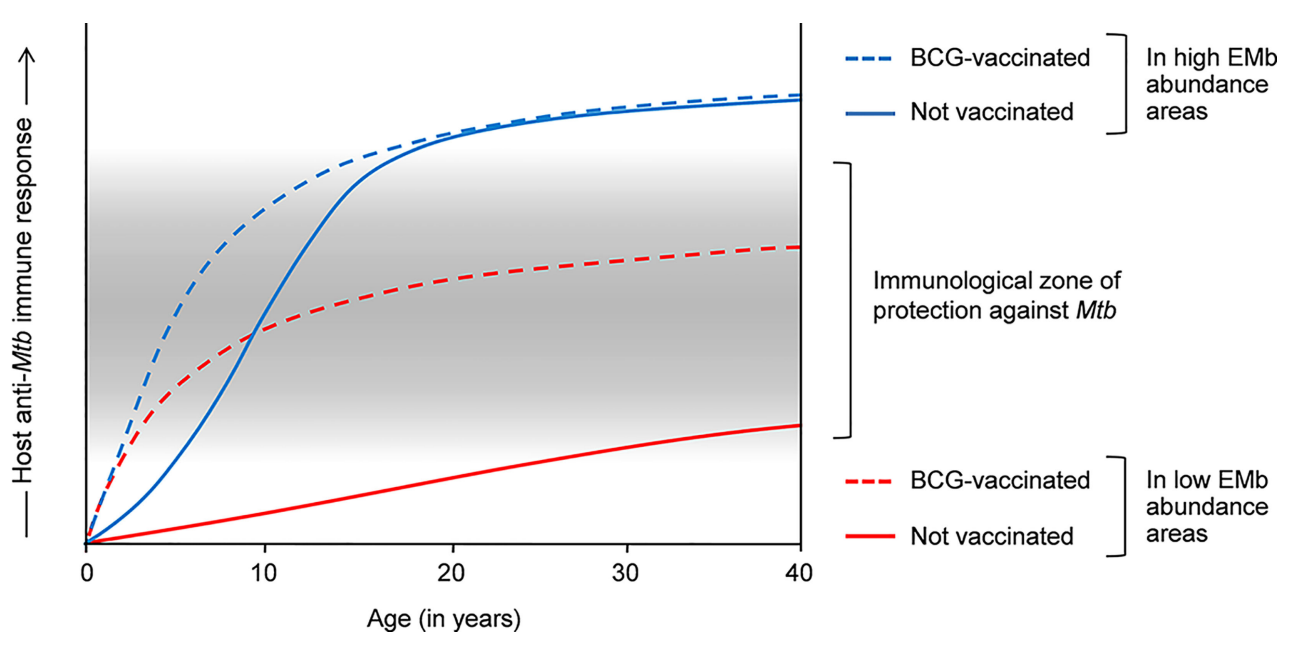

FIGURE 1 | Effects of environmental mycobacteria (EMb) and BCG on the host response to Mtb and the cross-talk thereof. Host response to Mtb is complex and heterogeneous. Infants and young children have a poorly developed immune system, which is incompetent in containing Mtb infection. BCG vaccination in these people promotes $T_{H} 1$ responses to $M t b$, resulting in the effective containment of the bacilli and significant protection against childhood TB (dashed blue and red lines). Owing to the presence of cross-reactive antigens, EMb also activate a degree of immunity against Mtb and therefore, confer some protection against childhood TB (blue line). However, frequent EMb exposure leads to the aggravation of anti-Mtb immunity in immunocompetent adults, which drives TB pathogenesis and results in higher incidence of pulmonary TB in the EMb-abundant areas (normal blue line). Similar aggravation of anti-Mtb immunity occurs in BCG-vaccinated adults in the EMb-abundant areas and leads to higher incidence of adult pulmonary TB and low efficacy of BCG in these places (dashed blue line). On the other hand, owing to low EMb exposure, BCG-mediated immunity against Mtb is not substantially modulated in the adult inhabitants in the areas of lower EMb abundance (dashed red line). Accordingly, vaccinated adults exhibit a moderately intense anti-Mtb immune response, which confers significant protection against adult pulmonary TB in these areas. 